\title{
Slope invariant $T$-linear resistivity from local self-energy
}

\author{
Peter Cha, ${ }^{1}$ Aavishkar A. Patel $\odot,{ }^{2,3}$ Emanuel Gull, ${ }^{4}$ and Eun-Ah Kim ${ }^{1}$ \\ ${ }^{1}$ Department of Physics, Cornell University, Ithaca, New York 14853, USA \\ ${ }^{2}$ Department of Physics, Harvard University, Cambridge, Massachusetts 02138, USA \\ ${ }^{3}$ Department of Physics, University of California Berkeley, Berkeley, California 94720, USA \\ ${ }^{4}$ Department of Physics, University of Michigan, Ann Arbor, Michigan 48109, USA
}

(Received 28 October 2019; revised 10 July 2020; accepted 16 July 2020; published 17 September 2020; corrected 11 December 2020)

\begin{abstract}
A theoretical understanding of the enigmatic linear-in-temperature $(T)$ resistivity, ubiquitous in strongly correlated metallic systems, has been a long sought-after goal. Furthermore, the slope of this robust $T$-linear resistivity is also observed to stay constant through crossovers between different temperature regimes: a phenomenon we dub "slope invariance." Recently, several solvable models with $T$-linear resistivity have been proposed, putting us in an opportune moment to compare their inner workings in various explicit calculations. We consider two strongly correlated models with local self-energies that demonstrate $T$ linearity: a lattice of coupled Sachdev-Ye-Kitaev models and the Hubbard model in single-site dynamical mean-field theory. We find that the two models achieve $T$ linearity through distinct mechanisms at intermediate temperatures. However, we also find that these mechanisms converge to an identical form at high temperatures. Surprisingly, both models exhibit "slope invariance" across the two temperature regimes. Thus not only do we reveal some of the diversity in the theoretical inner workings that can lead to $T$-linear resistivity, but we also establish that different mechanisms can result in "slope invarance."
\end{abstract}

DOI: 10.1103/PhysRevResearch.2.033434

\section{INTRODUCTION}

The mysterious incoherent metallic states with $T$-linear resistivity $\left(\rho_{\mathrm{DC}} \propto T\right)$ seen in many strongly correlated materials [1-3] have long puzzled researchers, as such a temperature dependence is inaccessible from Fermi liquid theory [4]. Particularly remarkable is the fact that the slope $d \rho_{\mathrm{DC}} / d T$ remains constant as $T$ varies over two or three orders of magnitude, while the temperature shoots through multiple crossover energy scales: we dub this phenomenon "slope invariance.” Recent progress [5-10] in solvable strongly interacting models that yield $T$-linear resistivity and through computational and experimental quantum simulation [11] have injected renewed enthusiasm and hope into the community. Given that "solvability" requires unrealistic limits, however, theoretical insight into the common or unique mechanisms of the $T$-linear resistivity obtained in these models is much needed in making contact with experimental observations.

The unusual feature of local self-energy is at play in two of the most studied microscopic models with $T$-linear resistivity: lattice models of coupled Sachdev-Ye-Kitaev (SYK) quantum dots [5-8] and its earlier incarnation of a doped random-bond Heisenberg model [12] and the Hubbard model in single-site dynamical mean-field theory (DMFT) $[11,13,14]$. Moreover, in both models, exact nontrivial self-consistency equations

Published by the American Physical Society under the terms of the Creative Commons Attribution 4.0 International license. Further distribution of this work must maintain attribution to the author(s) and the published article's title, journal citation, and DOI. enable an explicit computation. Yet no comparative study has been carried out to our best knowledge. In fact, these models have remained largely only of theoretical interest because $T$-linear resistivity in these models is reached only at temperatures above certain crossover scales that are too high for solidstate systems. However, in a recent emulation of the Hubbard model using ultracold atomic gases [11], $T$-linear resistivity was observed at "intermediate temperatures" $(t<T<U$, where $t$ and $U$ are the hopping and interaction energies, respectively) accessible in model calculations. Furthermore, "slope invariance" is also evident in the data. This motivates a fresh consideration of solvable models with $T$-linearity at intermediate and high temperatures seeking an insight into slope invariance. In this article we compare the inner workings of transport in two models with local self-energy, coupled SYK and DMFT, in two temperature ranges: intermediate $(t \ll T \ll U)$ and high temperature $(t \ll U \ll T)$. We find distinct mechanisms at intermediate temperatures converging to a unifying picture at high temperature. Further we note how the unifying picture ties to "slope invariance."

For both models of interest, vertex corrections to the conductivity vanish $[6,7,15]$. Hence the DC conductivity can be compactly written in the spectral representation of the Kubo formula

$$
\sigma_{\mathrm{DC}}=2 \pi \int d \epsilon \phi(\epsilon) \int \frac{\beta d \omega A(\epsilon, \omega)^{2}}{4 \cosh ^{2}(\beta \omega / 2)},
$$

where $\epsilon=\epsilon(\mathbf{k})$ is the band energy at momentum $\mathbf{k}, \phi(\epsilon)=$ $\sum_{\mathbf{k}}{\frac{\partial \epsilon(\mathbf{k})}{\partial k_{x}}}^{2} \delta[\epsilon-\epsilon(\mathbf{k})]$ is the transport function, and $A(\epsilon, \omega)=$ $-\frac{1}{\pi} \operatorname{Im} G(\epsilon, \omega)$ is the spectral function on the lattice, normalized to $\int d \omega A(\epsilon, \omega)=1$. Hence different mechanisms of 
$T$-linear resistivity arise from different functional forms of the spectral function.

\section{INTERMEDIATE TEMPERATURES}

We first review recent results finding $T$-linear resistivity in coupled SYK models [5-8]. These models are the best understood among the solvable models with $T$-linear resistivity, and they may be realizable in multiorbital systems $[16,17]$. In the SYK models, disorder averaging enforces the constraint that the self-energy of conduction electrons is local $\Sigma(\overrightarrow{\mathbf{k}}, i \omega) \equiv \Sigma(i \omega)$, as long as the coupling between SYK dots is perturbative. The self-consistent Dyson equations then arise in closed form as the saddle-point equations of a large- $N$ limit (where each SYK dot consists of $N$ flavors of fermions), in which the self-energy diagrams are truncated at second order. At intermediate and high temperatures, the hopping between lattice sites is perturbative, so that the lattice Green's function is also local $G(\overrightarrow{\mathbf{k}}, i \omega) \equiv G(i \omega)$ and the Dyson equations take the general form

$$
\begin{aligned}
G(i \omega)^{-1} & =i \omega+\mu-\Sigma(i \omega), \\
\Sigma(\tau) & =-U^{2} G(\tau)^{q / 2} G(-\tau)^{q / 2-1} .
\end{aligned}
$$

Here $q$ is the fermionic degree of the disordered SYK interaction in the Hamiltonian and $\mu$ is the chemical potential. In the intermediate-temperature regime, where $T \ll U$, the $i \omega$ term in the first line can be ignored. At half-filling, these equations then have the solution $G(\omega) \propto-i \beta^{1-2 / q} g_{q}(\beta \omega)$, where we have defined for convenience $g_{q}(x)=\Gamma(1 / q-$ $i x / 2 \pi) / \Gamma(1-1 / q-i x / 2 \pi)$.

Due to the local spectral function, the Kubo expression (1) then further simplifies to $\sigma_{\mathrm{DC}} \propto \int \frac{\beta d \omega A(\omega)^{2}}{4 \cosh ^{2}(\beta \omega / 2)}$. Hence,

$$
\sigma_{\mathrm{DC}} \propto t^{2} \beta^{2(1-2 / q)} \int d x \frac{\left[g_{q}(x)\right]^{2}}{\cosh ^{2}(x / 2)} \propto t^{2} \beta^{2(1-2 / q)},
$$

given the solution to the Dyson equation. This streamlined derivation makes clear that $T$-linear resistivity requires $q=4$ $[7,18]$, in which case the complete expression for the conductivity at intermediate temperatures $t^{2} / U \ll T \ll U$ is

$$
\sigma_{\mathrm{DC}}=\frac{t^{2} \beta \sqrt{\pi}}{2 U \sqrt{\cosh 2 \pi \mathcal{E}}}
$$

$[5,6]$, where $\mathcal{E}$ is a function solely of the fermion filling that vanishes at half-filling $[19,20]$. Two features of this calculation offer clarifying insight: First, the SYK spectral function is a scaling function of the dimensionless parameter $\beta \omega$ and extends broadly over the entire frequency range below the UV cutoff $|\omega|<U$ (Appendix A 2). Second, although local selfenergy is a generic feature of SYK models, $T$-linear resistivity in the intermediate-temperature range is a consequence of the particular scaling exponent of the local Green's function within the "quantum dots" for $q=4$. The same mechanism was found earlier in a doped random Heisenberg model in Ref. [12].

Now we turn to the DMFT study of the repulsive $U$ Hubbard model on the two-dimensional square lattice:

$$
H=-t \sum_{\langle i, j\rangle, \sigma} c_{i \sigma}^{\dagger} c_{j \sigma}+U \sum_{i} n_{i \uparrow} n_{i \downarrow}-\mu \sum_{i \sigma} n_{i \sigma} .
$$

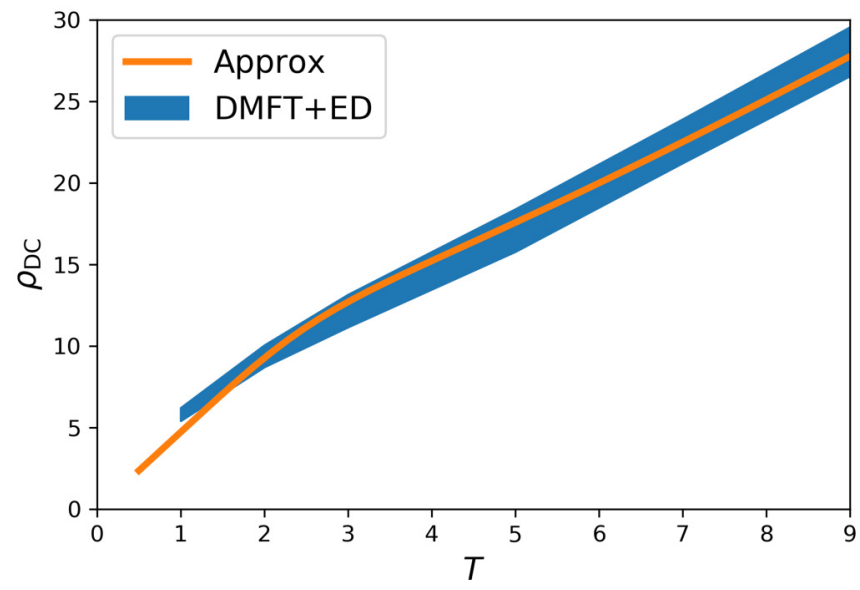

FIG. 1. Resistivity $\rho_{\mathrm{DC}}$ as a function of temperature, in singlesite DMFT with $U=12$ at electron filling $n=0.825$, using an ED impurity solver with $n_{s}=8$ total sites. Vertical spread of the blue curve comes from varying the ED broadening parameter $\eta=$ 0.01-0.08. Orange curve displays the approximation scheme of Eq. (7).

In DMFT, we first integrate out all sites except one to reduce the problem to a local impurity model. Then the infinite-dimension approximation imposes a self-consistency constraint where the bare propagator of the impurity model is determined by the fully dressed two-point function computed within the impurity model. Lacking an additional large- $N$ parameter of SYK, a closed-form Dyson equation is not available, however. Hence the impurity model is solved computationally. Despite intense efforts at computational studies of the model and its extensions and observations of $T$-linear resistivity upon certain approximations [13,14,21-23], new interest in models with local self-energies warrants a close contextual look at the DMFT solutions to the Hubbard model. Here we carry out single-site DMFT using an exact diagonalization (ED) impurity solver that frees us from the need of analytic continuation.

We explored the range of $7.5<U<12$ and $0.2<T<$ 9 in units of hopping $t$, with the electron density per site $n=0.825$. In Fig. 1 we plot a representative result. Here the width of the band represents the dependence of $\rho_{\mathrm{DC}}(T)$ on the ED broadening parameter $\eta[11,24]$. The resulting curve is clearly linear in the intermediate-temperature range $t \ll T \ll U[25]$.

In search of analytic insight into $T$-linear resistivity at intermediate temperatures $t \ll T \ll U$, we examine the lattice spectral function $A(\epsilon, \omega)$ in detail for a suitable analytic ansatz. The spectral function is largely $T$-independent at the intermediate temperatures shown and consists of a lower and an upper band of widths $\sim t$ that are separated in frequency by $\approx U$ (see Fig. 2). Hence we approximate the DMFT spectral function using the following ansatz:

$$
A(\epsilon, \omega)=a_{l} h\left(\omega ; \omega_{l}, \eta_{l}\right)+a_{u} h\left(\omega ; \omega_{u}, \eta_{u}\right),
$$

where the lower and upper bands $A_{l, u}(\epsilon, \omega)$ have weights $a_{l, u}$, satisfying $a_{l}+a_{u}=1$, and are localized in frequencies at $\omega_{l, u}$ with widths $\eta_{l, u} \ll T$. For simplicity, we model each band $h(\omega)$ by a normalized Gaussian, centered at $\omega_{l, u}$ 


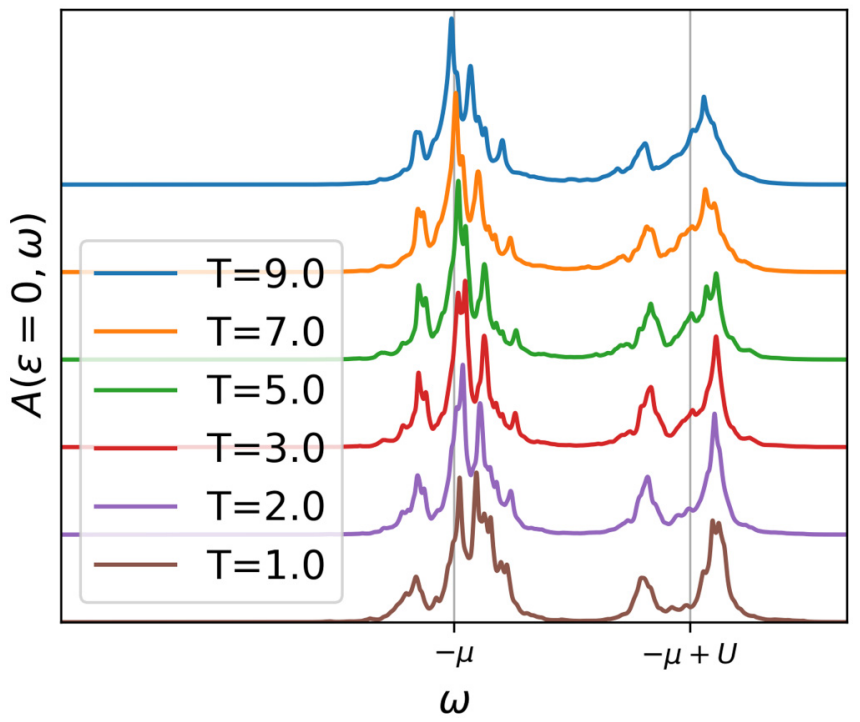

FIG. 2. Lattice spectral function $A(\epsilon=0, \omega)$ vs frequency $\omega$, with $U=12$ at electron filling $n=0.825$, using ED impurity solver with $n_{s}=8$ and $\eta=0.08$. Frequency $\omega$ has been shifted by the chemical potential.

with standard deviation $\eta_{l, u}$. Then the parameters for the lower band $a_{l}, \omega_{l}, \eta_{l}$ are fitted to the DMFT spectral function by $\int d \omega A_{l}(\epsilon, \omega)=a_{l}, \int d \omega \omega A_{l}(\epsilon, \omega)=a_{l} w_{l}$, and $\int d \omega\left[A_{l}(\epsilon, \omega)\right]^{2}=1 / 2 \sqrt{\pi} a_{l}^{2} / \eta_{l}$, and likewise for the upper band parameters [26]. The parameters depend on $\epsilon$ but are independent of $T$. With this ansatz, we arrive at a simplified explicit expression for DC conductivity (see Appendix A 1 for a detailed derivation)

$$
\sigma_{\mathrm{DC}}=\beta \frac{C_{l}}{4 \cosh ^{2}(\beta \mu / 2)}+\frac{C_{u}}{4 \cosh ^{2}[\beta(U-\mu) / 2]},
$$

where $C_{l, u} \equiv \sqrt{\pi} \int d \epsilon \phi(\epsilon) a_{l, u}^{2} / \eta_{l, u}$. In the intermediatetemperature range, Eq. (7) yields $T$-linear resistivity by the first term in the parentheses approaching a constant $(\mu \propto T)$ and second term vanishing $(T \ll U)$. We note that similar approximations were employed in previous works [27,28], which yielded similar expressions for conductivity.

Figure 1 demonstrates that the ansatz Eq. (7) (shown in orange) captures the DMFT results for resistivity excellently over the whole temperature range. This fit reveals that key mechanism for $T$-linear resistivity in DMFT is the broadening of the spectral function by a $T$-independent scattering rate $1 / \tau$ controlled by a hopping scale $\sim t$. Such a highly localized spectral function leads to $\sigma_{\mathrm{DC}} \propto \tau / T$ from a linearization of the Fermi factor in the Kubo formula. We suspect the same mechanism is at play for $t \ll U$ modified Hubbard model studied by Mousatov et al. [9] where the broadening in that case is set by a longer-ranged repulsion $V$. This is in contrast to the inner workings of coupled SYK, where the spectral function is a broad, singly peaked scaling function of $\beta \omega$ and $T$-linear resistivity is specific to $q=4$ (Appendix A 2).

Armed with the above analytic insight, we now turn to the Lorenz ratio $L=\kappa / \sigma T$. The Wiedemann-Franz law with $L_{W F}=\pi^{2} / 3$ is a well-known property of a Fermi liquid with elastic scattering. Although it can be violated even in a Fermi liquid in the presence of inelastic scattering [29-31], it is nevertheless a useful quantity to evaluate. The thermal and electrical conductivities can be expressed in terms of kinetic coefficients

$$
L_{n}=2 \pi \int d \epsilon \phi(\epsilon) \int \frac{\beta d \omega(\beta \omega)^{n} A(\epsilon, \omega)^{2}}{4 \cosh ^{2}(\beta \omega / 2)}
$$

as $\sigma=L_{0}$ and $\kappa=T\left(L_{2}-\left(L_{1}\right)^{2} / L_{0}\right)$. Once again employing our approximate spectral function (6), we find the violation of the Wiedemann-Franz law with the Lorenz ratio vanishing as a power law in temperature for $t \ll T$. In contrast, the coupled SYK model of Ref. [5] violates the Wiedemann-Franz law only through modification of the Lorenz ratio $L=0.375 \times$ $L_{\mathrm{WF}}[5,6]$ in the intermediate-temperature regime with $T$ linear resistivity.

\section{HIGH-TEMPERATURE LIMIT}

We now consider the high-temperature regime, where $T$ is the largest scale in the problem. Resistivity at high temperature is often overlooked, as all Hamiltonians with a bounded spectrum of states are known to have $T$-linear resistivity in this temperature regime $[32,33]$. This argument is useful from a formal perspective but offers little insight into $T$-linear resistivity at intermediate temperatures or the lack of slope change across the crossover $T \sim U$. In this "weak coupling" limit, we approximate single-site DMFT using self-consistent second-order perturbation theory (GF2) [34-37]. Unlike bare second-order perturbation theory, GF2 is $\Phi$-derivable [38] and therefore thermodynamically consistent and symmetry conserving $[39,40]$, implying that thermodynamic relations and conservation laws are intrinsically satisfied. We find, within this high-temperature limit, that the DMFT equations become identical to the self-consistency equations of $q=4$ SYK!

To see the convergence note that the self-energy $\Sigma(i \omega)$ is the sum of a tadpole diagram and a sunset diagram in GF2, i.e., $\Sigma(i \omega)=\Sigma^{(1)}(i \omega)+\Sigma^{(2)}(i \omega)$, where $\Sigma^{(1)}(i \omega)=$ $U G\left(\tau=\beta^{-}\right)=U n / 2 \quad$ and $\quad \Sigma^{(2)}(\tau)=-U^{2} G(\tau)^{2} G(-\tau)$. Now the Dyson equations take a closed form:

$$
\begin{aligned}
G(i \omega)^{-1} & =i \omega+\mu_{\text {eff }}-\Sigma^{(2)}(i \omega), \\
\Sigma^{(2)}(\tau) & =-U^{2} G(\tau)^{2} G(-\tau),
\end{aligned}
$$

which are identical to the $q=4$ SYK equations (2), with a shifted chemical potential $\mu_{\text {eff }}=\mu-n U / 2$. This discovery allows us to simultaneously treat single-site DMFT and $q=4$ SYK.

Interestingly, with $T \gg U$, we find, by numerical solution of the real-time version of (9), that the self-energy is temperature-independent and Gaussian to a very good approximation (Fig. 3). Using a high-temperature expansion in imaginary-time [41] and the "maximum entropy" ansatz for analytic continuation [33], we further determine, for $\mu_{\text {eff }}=0$,

$$
-\frac{1}{\pi} \operatorname{Im} \Sigma(\omega) \approx \frac{U}{2 \pi} \sqrt{\frac{\pi}{6}} \exp \left(-\frac{2 \omega^{2}}{3 U^{2}}\right) .
$$

For $\mu_{\text {eff }} \neq 0$ we find a similar function with its peak shifted to $\omega=\mu_{\text {eff. }}$. A Gaussian self-energy of width $\sim U$ leads to a spectral function also of width $\sim U$. In the high-temperature 


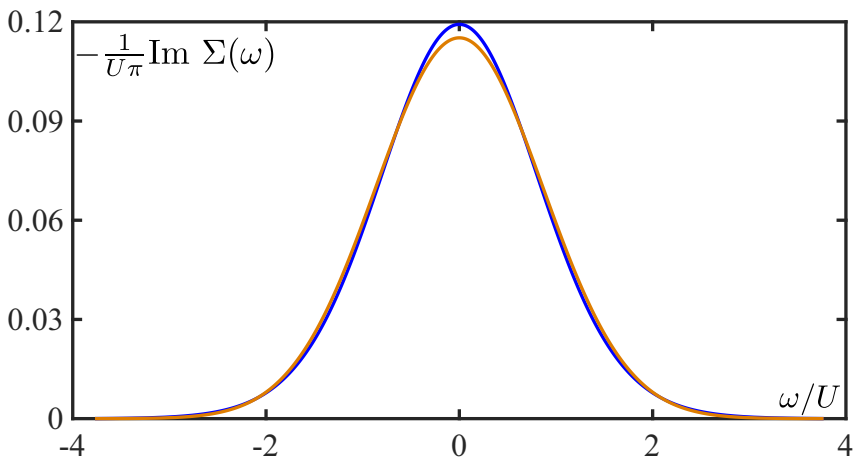

FIG. 3. Comparison of the numerically obtained self-energy (blue) in for the $q=4 \mathrm{SYK}$ model in the high-temperature regime with a Gaussian ansatz (orange) for analytically continuing a hightemperature expansion (10). Here $\beta U=0.01$, and the system is at half-filling.

regime $U \ll T$, this leads to $T$-linear resistivity in the same manner as (7), but with only one band at $-\mu_{\text {eff }}$.

The divergence of the two models at intermediate temperatures is visible in how the asymptotic form of selfenergy responds to the lowering of temperature. In DMFT, the self-energy becomes increasingly sharply peaked at lower temperatures. This trend is visible in Fig. 4, which displays the self-energy in DMFT across a range of intermediate temperatures for $U=12$ and $n=0.825$. We have also plotted a Gaussian fit to the primary peak in dashed lines in Fig. 4. Gaussian self-energy in DMFT at intermediate temperatures was suggested in Ref. [33] by maximum entropy analytic continuation. In sharp contrast, the selfenergy in $q=4$ coupled SYK undergoes a dramatic change in form, departing from the Gaussian form at high temperatures to $-\operatorname{Im} \Sigma(\omega \ll U) \sim \max (\sqrt{U T}, \sqrt{U \omega})$ (Appendix A 2).

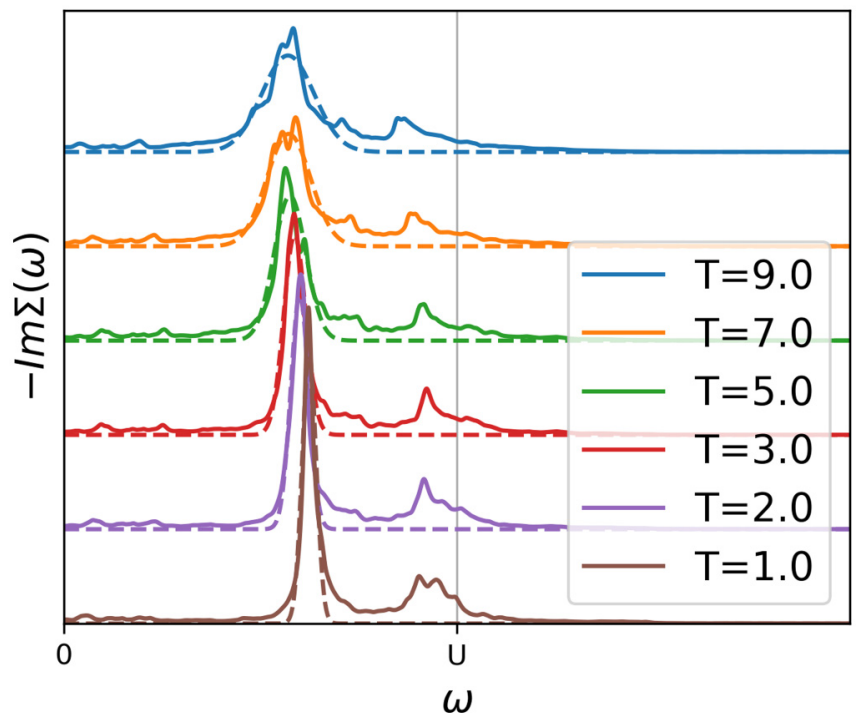

FIG. 4. Imaginary self-energy $-\operatorname{Im} \Sigma(\omega)$ vs real frequency $\omega$, from DMFT with $U=12$ at electron filling $n=0.825$, using ED impurity solver with $n_{s}=8$ and $\eta=0.08$. Dashed line plots a Gaussian fit to the central peak of self-energy at each temperature. Frequencies have been shifted by $\mu_{\text {eff }}$.

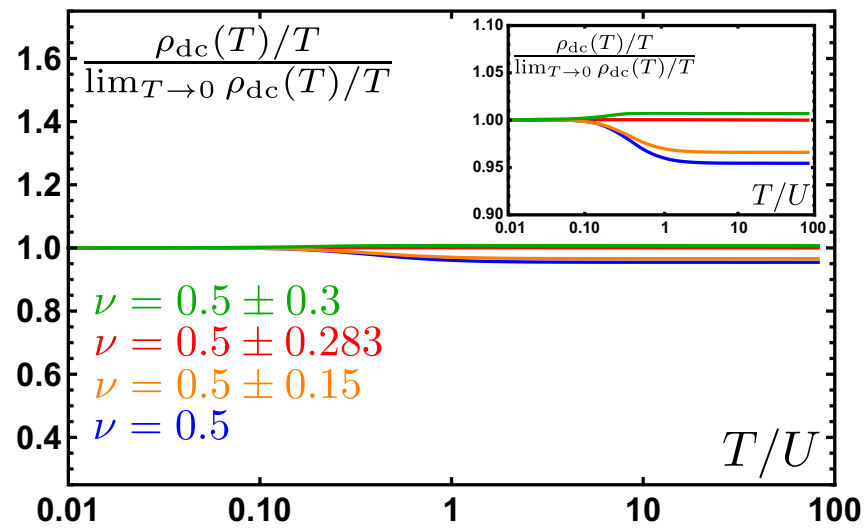

FIG. 5. Normalized change in slope of linear-in- $T$ resistivity for the $q=4$ SYK chain with infinitesimally weak quadratic hopping at different fillings $v$. The inset shows the same plot with the $y$ axis magnified.

Surprisingly, despite this qualitative shift in the single-particle properties, the slope change in resistivity $d \rho_{\mathrm{DC}} / d T$ remains negligible across $T \sim U$ for all numerically accessible values of the filling [42] and actually goes to zero within numerical tolerances at fillings $\approx 0.5 \pm 0.283$ (Fig. 5).

The contrasting forms of the DMFT and SYK self-energies below the high-temperature limit can be traced to the validity of GF2. In the SYK incoherent metal, GF2 is exact independently of temperature because the large number of degrees of freedom $N$, with only disordered interactions among them, explicitly makes all self-energy diagrams beyond second order nonthermodynamic. This exact truncation cannot be done in single-site DMFT where each local electron degree of freedom has only self-interactions and there is no such large$N$ limit. Thus GF2 is approximately valid in DMFT only in the high-temperature limit where interactions are effectively perturbative. As temperature is lowered across the crossover scale $U$, higher order processes of $O\left[(U / T)^{3}\right]$ become relevant and lead to a breakdown of GF2.

\section{DISCUSSION}

At intermediate temperatures $T \ll U$, we observed that single-site DMFT achieves $T$-linear resistivity by grouping the spectrum of states into two narrow bands of widths far below the interaction scale $U$. In the same temperature regime, SYK lattice models have a broad spectrum that extends over the entire range $|\omega| \lesssim U$ and give $T$-linear resistivity for models with $q=4$, for which $A(\omega) \sim 1 / \sqrt{U \omega}$ for $T \ll \omega \ll U$. Although the intermediate-temperature mechanisms behind $T$-linear resistivity in these models are seemingly unrelated, we have made the surprising observation that the models seem to converge to an identical form at high temperature $T \gg U$. We further observe that the $q=4$ SYK model shows almost no slope change in its $T$-linear resistivity across the crossover scale $T \sim U$ (Fig. 5).

Our finding of "slope invariance" in lattice SYK despite major changes in the single-particle spectral properties is remarkably reminiscent of observations made in strongly correlated materials. On the other hand, the slope in the 
two temperature regimes have distinct doping dependences in DMFT and slope invariance requires fine tuning in doping [33]. However, the crossover temperature is suppressed from the naïve $\sim U$ to $\sim U / \log \left(1 / \delta^{2}\right)$ at small dopings [33] due to temperature dependence of chemical potential. This accounts for the observation that the change in slope in Fig. 1 occurs at $T \approx 2=U / 6$, as opposed to at $T \approx U$. Furthermore, the slopes in the two temperature regimes differ by less than $11 \%$ for all large enough doping $d>0.33$ (see Appendix A 3). The renomalized crossover scale can push the slope change outside of range of observation which may explain the featureless $T$-linear resistivity reported in Ref. [9]. Finally, the change in $T$ dependence of chemical potential that drives the slope change in $T$-linear resistivity is also responsible for a slope change in inverse compressibility $\chi^{-1}$ (see Appendix A 3), which can further complicate any inference on diffusivity from the Nernst-Einstein relation $\sigma_{\mathrm{DC}}=\chi \mathcal{D}$ and calculation of $\chi[9,21,43]$.

\section{ACKNOWLEDGMENTS}

We thank Erez Berg, Antoine Georges, David Huse, Srinivas Raghu, and Subir Sachdev for helpful discussions. P.C. and E.-A.K. were supported by the U.S. Department of Energy, Office of Basic Energy Sciences, Division of Materials Science and Engineering under Award DE-SC0018946 and the W. M. Keck Foundation. A.A.P. was supported by the U.S. Department of Energy under Grant No. DE-SC0019030, and by the Miller Institute for Basic Research in Science. E.G. acknowledges sabbatical support by the Center for Computational Quantum Phsyics of the Flatiron Institute. This work was initiated at Aspen Center for Physics, which is supported by National Science Foundation grant PHY-1607611.

\section{APPENDIX}

\section{A. Detailed derivation of conductivity}

In this section we derive the expression for conductivity (7) from the Kubo formula

$$
\sigma_{\mathrm{DC}}=2 \pi \int d \epsilon \phi(\epsilon) \int \frac{\beta d \omega A(\epsilon, \omega)^{2}}{4 \cosh ^{2}(\beta \omega / 2)} .
$$

From the DMFT spectral function in Fig. 2, we employ the ansatz of (6)

$$
A(\epsilon, \omega)=a_{l} h\left(\omega ; \omega_{l}, \eta_{l}\right)+a_{u} h\left(\omega ; \omega_{u}, \eta_{u}\right),
$$

where the lower and upper bands $A_{l, u}(\epsilon, \omega)$ have weights $a_{l, u}$, satisfying $a_{l}+a_{u}=1$, and are localized in frequencies at $\omega_{l, u}$ with widths $\eta_{l, u} \ll T$. Note that the ansatz parameters $a_{l, u}$, $\omega_{l, u}$, and $\eta_{l, u}$ can depend on the band energy $\epsilon$.

We can now scale out $\beta$ by changing the variable of integration $x \equiv \beta \omega$. The spectral function in terms of $x$ is

$$
A(\epsilon, x)=\beta a_{l} h_{x}\left(x ; \beta \omega_{l}, \beta \eta_{l}\right)+a_{u} h_{x}\left(x ; \beta \omega_{u}, \beta \eta_{u}\right) .
$$

If the spectral function consists of widely separated narrow peaks $\eta_{l, u} \ll\left|\omega_{u}-\omega_{l}\right|$, then interband processes will be suppressed

$$
A(\epsilon, x)^{2} \approx \beta^{2} a_{l}^{2} h_{x}\left(x ; \beta \omega_{l}, \beta \eta_{l}\right)^{2}+a_{u}^{2} h_{x}\left(x ; \beta \omega_{u}, \beta \eta_{u}\right)^{2} .
$$

The $x$ integral can now be carried out explicitly. We focus on the lower band for brevity but the calculation for the upper band is identical.

$$
\begin{aligned}
\int \frac{\beta d \omega A(\epsilon, \omega)^{2}}{4 \cosh ^{2}(\beta \omega / 2)} & =\int \frac{d x \beta^{2} a_{l}^{2} h_{x}\left(x ; \beta \omega_{l}, \beta \eta_{l}\right)^{2}}{4 \cosh ^{2}(x / 2)} \\
& =\frac{\beta^{2} a_{l}^{2}}{4 \cosh ^{2}\left(\beta \omega_{l} / 2\right)} \frac{1}{2 \sqrt{\pi} \beta \eta_{l}} \\
& =\frac{1}{2 \sqrt{\pi}} \frac{\beta a_{l}^{2}}{4 \eta_{l} \cosh ^{2}\left(\beta \omega_{l} / 2\right)} \\
& \approx \frac{1}{2 \sqrt{\pi}} \frac{\beta a_{l}^{2}}{4 \eta_{l} \cosh ^{2}(\beta \mu / 2)},
\end{aligned}
$$

where in the final line we make the observation that $\mid \omega_{l}+$ $\mu \mid \ll T$ for all $\epsilon$. The lower band contribution to the conductivity can thus be written

$$
\sigma_{\mathrm{DC}, l}=\beta \frac{C_{l}}{4 \cosh ^{2}(\beta \mu / 2)},
$$

where we have defined $C_{l} \equiv \sqrt{\pi} \int d \epsilon \phi(\epsilon) a_{l, u}^{2} / \eta_{l, u}$. Summing the contribution from the upper band, we arrive at the expression for conductivity (7).

\section{B. Comparative Plot}

In Fig. 6 we show typical plots of the self-energies and spectral functions in the two temperature regimes from the DMFT calculation and the coupled SYK calculation.

\section{Compressibility}

Recent works have proposed looking for insight into $T$ linear resistivity by studying compressibility $\chi$ and diffusivity $\mathcal{D}$, related to transport by the Nernst-Einstein relation $\sigma_{\mathrm{DC}}=\chi \mathcal{D}$, at intermediate and high temperatures. $[9,21,43]$. We obtain compressibility in DMFT using continuous-time interaction-expansion QMC as the impurity solver. We computed the DMFT solution for multiple values of $\mu$ near the value corresponding to $n=0.825$ and took the numerical derivative to find $\chi^{-1}=\frac{d \mu}{d n}$. No analytical continuation is necessary as $n=-2 G\left(\tau=\beta^{-}\right)$. We present the data from DMFT in Fig. 7.

To gain further insight, we summarize analytical results in the perturbative-hopping regime $t \ll T, U$, discussed in Refs. [28,33]. In this limit, the chemical potential $\mu$ and DC conductivity $\sigma_{\mathrm{DC}}$ can be calculated from the atomic Hubbard model, i.e., a Hubbard model with $t \rightarrow 0$. These expressions are

$$
\begin{aligned}
e^{\beta \mu} & =\frac{\sqrt{\delta^{2}+\left(1-\delta^{2}\right) y}-\delta}{(1+\delta) y} \equiv x, \\
\sigma_{\mathrm{DC}} & =\frac{t}{4 T} \frac{(1+\delta)^{2}}{x+1 / x+2}+\frac{(1-\delta)^{2}}{x y+1 / x y+2},
\end{aligned}
$$

where $\delta=1-n, y=e^{-U / T}$ and the second expression is valid up to dimensionless constants.

From these expressions, we can take further limits to study the compressibility $\chi$, DC conductivity $\sigma_{\mathrm{DC}}$, and 

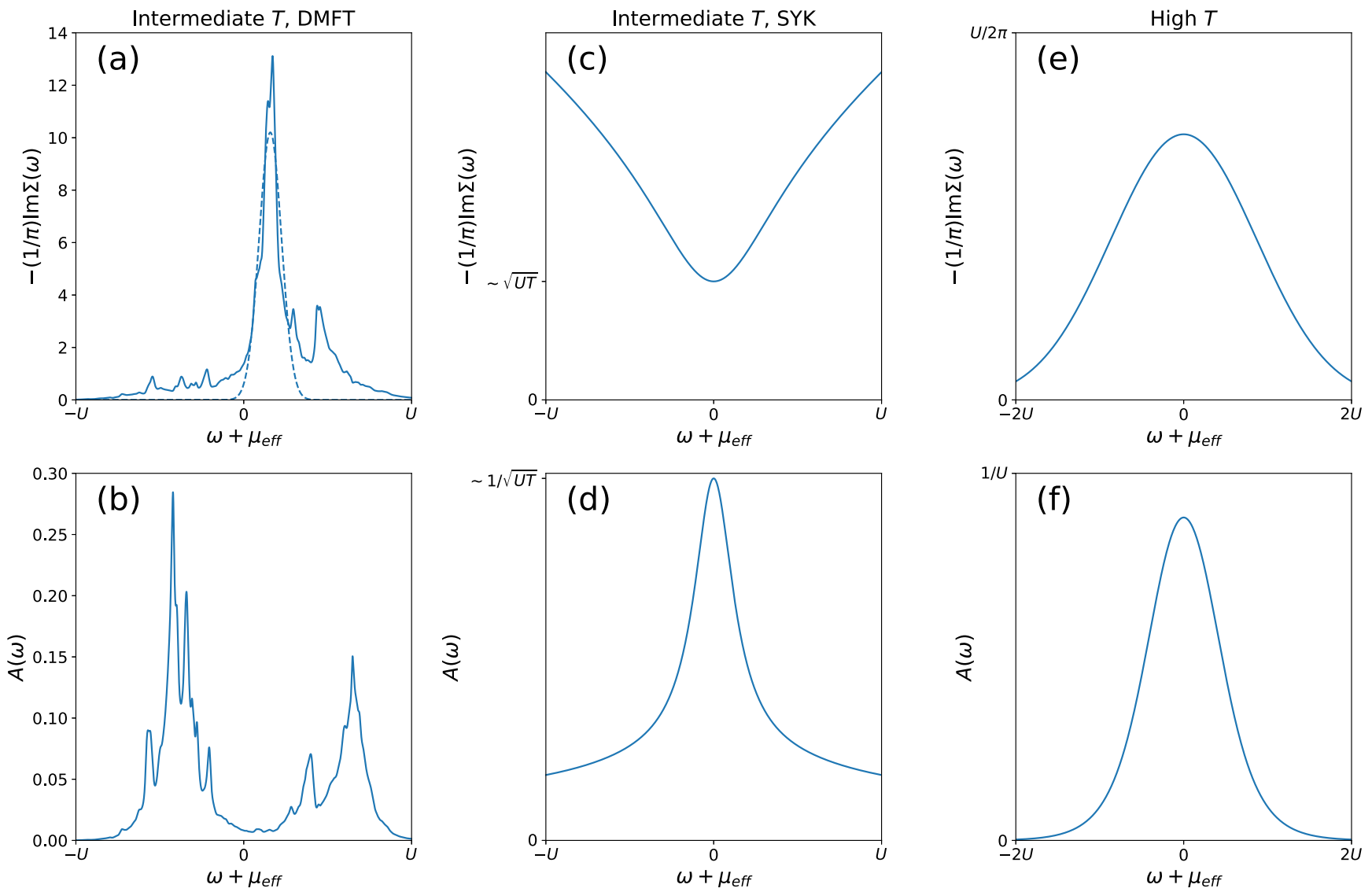

FIG. 6. Plots of self-energy (a), (c), (e) and spectral function (b), (d), (f) vs frequency shifted by $\mu_{e f f}$. The dashed line in (a) displays the gaussian fit of Fig. 4. The singly-peaked self-energy of DMFT at intermediate $T$ (a) contrasts with the self-energy of SYK also at intermediate $T$ (c), which has a local minimum at $\omega+\mu_{e f f}=0$, while the DMFT spectral function (b), which is highly localized in frequency, contrasts with the broad scaling form of the SYK spectral function (d). Despite these contrasting forms at intermediate temperature, the two models converge to the Gaussian self-energy form at high temperature (e), (f).

diffusivity $\mathcal{D} \equiv \sigma_{\mathrm{DC}} / \chi$ in the intermediate-temperature $t \ll$ $T \ll U$ or high-temperature $t \ll U \ll T$ limits. In the intermediate-temperature regime, we find

$$
\mu_{\text {int }}=T \log \frac{n}{2(1-n)},
$$

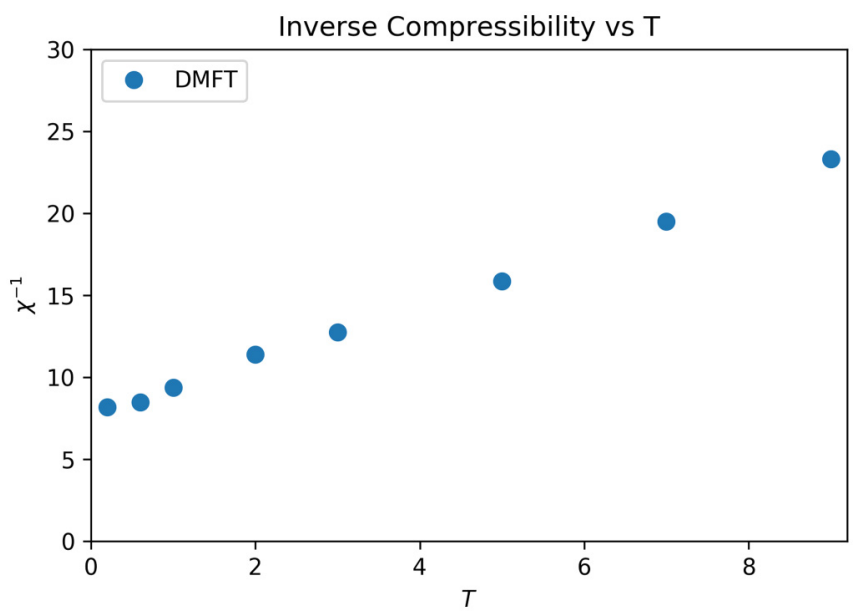

FIG. 7. Inverse compressibility $\chi^{-1}$ at $U=7.5, n=0.825$.

$$
\begin{aligned}
\chi_{\text {int }}^{-1} & =\frac{T}{n(1-n)}, \\
\rho_{\text {DC }, \text { int }} & =\frac{2 T}{n(1-n)}, \\
\mathcal{D}_{\text {int }}^{-1} & =2 .
\end{aligned}
$$

On the other hand, in the high-temperature regime, we find

$$
\begin{gathered}
\mu_{\text {high }}=T \log \frac{n}{2-n}, \\
\chi_{\text {high }}^{-1}=\frac{2 T}{n(2-n)}+\frac{U}{2}, \\
\rho_{\text {DC,high }}=\frac{8 T}{1-(1-n)^{4}}, \\
\mathcal{D}_{\text {high }}^{-1}=\frac{4}{1+(1-n)^{2}} .
\end{gathered}
$$

We remark that $\mathcal{D}$ saturates to different expressions in both regimes. As noted in the main text, the crossover between the two expressions is set by the change in the temperature dependence of the chemical potential, which follows from setting $y \ll \frac{\delta^{2}}{1-\delta^{2}}$. At small doping, the crossover temperature is therefore suppressed from the naïve $\sim U$ to $\sim U / \log ((1-$ $\left.\left.\delta^{2}\right) / \delta^{2}\right)$. 
[1] S. Martin, A. T. Fiory, R. M. Fleming, L. F. Schneemeyer, and J. V. Waszczak, Phys. Rev. B 41, 846 (1990).

[2] R. A. Cooper, Y. Wang, B. Vignolle, O. J. Lipscombe, S. M. Hayden, Y. Tanabe, T. Adachi, Y. Koike, M. Nohara, H. Takagi et al., Science 323, 603 (2009).

[3] J. A. N. Bruin, H. Sakai, R. S. Perry, and A. P. Mackenzie, Science 339, 804 (2013).

[4] Y. Werman, S. A. Kivelson, and E. Berg, njp Quantum Mater. 2, 7 (2016).

[5] X.-Y. Song, C.-M. Jian, and L. Balents, Phys. Rev. Lett. 119, 216601 (2017).

[6] A. A. Patel, J. McGreevy, D. P. Arovas, and S. Sachdev, Phys. Rev. X 8, 021049 (2018).

[7] D. Chowdhury, Y. Werman, E. Berg, and T. Senthil, Phys. Rev. X 8, 031024 (2018).

[8] A. A. Patel, M. J. Lawler, and E.-A. Kim, Phys. Rev. Lett. 121, 187001 (2018).

[9] C. H. Mousatov, I. Esterlis, and S. A. Hartnoll, Phys. Rev. Lett. 122, 186601 (2019)

[10] A. A. Patel and S. Sachdev, Phys. Rev. Lett. 123, 066601 (2019).

[11] P. T. Brown, D. Mitra, E. Guardado-Sanchez, R. Nourafkan, A. Reymbaut, C.-D. Hébert, S. Bergeron, A.-M. S. Tremblay, J. Kokalj, D. A. Huse, P. Schauß, and W. S. Bakr, Science 363, 379 (2018).

[12] O. Parcollet and A. Georges, Phys. Rev. B 59, 5341 (1999).

[13] X. Deng, J. Mravlje, R. Žitko, M. Ferrero, G. Kotliar, and A. Georges, Phys. Rev. Lett. 110, 086401 (2013).

[14] J. Kokalj, Phys. Rev. B 95, 041110(R) (2017).

[15] A. Georges, G. Kotliar, W. Krauth, and M. J. Rozenberg, Rev. Mod. Phys. 68, 13 (1996).

[16] P. Werner, A. J. Kim, and S. Hoshino, Europhys. Lett. 124, 57002 (2018).

[17] N. Tsuji and P. Werner, Phys. Rev. B 99, 115132 (2019).

[18] $T$-linearity can be achieved for other values of $q$ by changing the fermionic degree of the hopping term [8]. For example, with $p$-fermion hopping

$$
\sum t_{i_{1} \ldots j_{p}} c_{i_{1}}^{\dagger} \ldots c_{i_{p}}^{\dagger} c_{j_{1}} \ldots c_{j_{p}}
$$

the expression for conductivity is modified as

$$
\begin{aligned}
\sigma_{\mathrm{DC}} & =2 \pi \int d \omega d \omega_{1} \ldots d \omega_{2 p-2} \frac{\beta A(\omega)^{2 p}}{\cosh ^{2}(\beta \omega / 2)} \\
& \propto \beta^{2(1-2 p / q)}
\end{aligned}
$$

showing that $T$-linearity is achieved when $q=4 p$.

[19] R. A. Davison, W. Fu, A. Georges, Y. Gu, K. Jensen, and S. Sachdev, Phys. Rev. B 95, 155131 (2017).

[20] The conductivity crosses over from (4) to Fermi-liquid like $1 / T^{2}$ behavior in the "low-temperature" regime of $T \ll t^{2} / J$ $[5,7,12]$. However, we always focus on a higher temperature range.

[21] E. W. Huang, R. Sheppard, B. Moritz, and T. P. Devereaux, Science 366, 987 (2019).

[22] J. Vučičević, D. Tanasković, M. J. Rozenberg, and V. Dobrosavljević, Phys. Rev. Lett. 114, 246402 (2015).
[23] J. Vučičević, J. Kokalj, R. Žitko, N. Wentzell, D. Tanasković, and J. Mravlje, Phys. Rev. Lett. 123, 036601 (2019).

[24] The lattice spectral function is defined by $A(\epsilon, \omega)=$ $-\lim _{\eta \rightarrow 0} \frac{1}{\pi} \operatorname{Im} G(\epsilon, \omega+i \eta)$. However, with finite bath sites, the spectral function is a sum of $\delta$ functions and conductivity is singular. In order to obtain a finite conductivity, a finite ED broadening parameter $\eta$ is introduced in the expression for the spectral function. We consider the results reliable when the conductivity does not vary much over a wide range of finite $\eta \ll 1$.

[25] Similar results are seen in the DMFT calculation of resistivity in Brown et al. [11], with the parameters $n=0.825, U=7.5$, $T<6$, with $n_{s}=6$.

[26] We have selected a Gaussian form for $h(\omega)$ in the text, but we note that the precise forms of each band are not crucial to the results. Using a different bounded function for $h(\omega)$ (e.g., Lorentzian) will affect only the proportionality constant in front of $a^{2} / \eta$ in the final fitting equation.

[27] G. Pálsson and G. Kotliar, Phys. Rev. Lett. 80, 4775 (1998).

[28] S. Mukerjee and J. E. Moore, Appl. Phys. Lett. 90, 112107 (2007).

[29] L. Taillefer, B. Lussier, R. Gagnon, K. Behnia, and H. Aubin, Phys. Rev. Lett. 79, 483 (1997).

[30] L. V. Pourovskii, J. Mravlje, A. Georges, S. I. Simak, and I. A. Abrikosov, New J. Phys. 19, 073022 (2017).

[31] A. Lavasani, D. Bulmash, and S. Das Sarma, Phys. Rev. B 99 , 085104 (2019).

[32] Consider the spectral representation of conductivity $\sigma(\omega)=$ $-\frac{\pi}{\omega} \sum_{n m}\left|\left\langle n\left|J_{x}\right| m\right\rangle\right|^{2} e^{-\beta E_{n}} e^{\beta E_{n m}}-1 \delta\left(\omega-E_{n m}\right)$ where $n, m$ labels the many-body eigenstates and $E_{n m}=E_{n}-E_{m}$. When $E_{n} \ll T$ for all $n$, the Boltzmann factors can be expanded to give a single factor of $\beta$ in the expression to leading order, which shows $T$-linear resistivity.

[33] E. Perepelitsky, A. Galatas, J. Mravlje, R. Žitko, E. Khatami, B. S. Shastry, and A. Georges, Phys. Rev. B 94, 235115 (2016).

[34] J. J. Phillips and D. Zgid, J. Chem. Phys. 140, 241101 (2014).

[35] S. Iskakov, A. A. Rusakov, D. Zgid, and E. Gull, Phys. Rev. B 100, 085112 (2019).

[36] M. Motta, D. M. Ceperley, G. K.-L. Chan, J. A. Gomez, E. Gull, S. Guo, C. A. Jiménez-Hoyos, T. N. Lan, J. Li, F. Ma, A. J. Millis et al. (Simons Collaboration on the Many-Electron Problem), Phys. Rev. X 7, 031059 (2017).

[37] A. A. Rusakov, S. Iskakov, L. N. Tran, and D. Zgid, J. Chem. Theory Comput. 15, 229 (2019).

[38] J. M. Luttinger and J. C. Ward, Phys. Rev. 118, 1417 (1960).

[39] G. Baym and L. P. Kadanoff, Phys. Rev. 124, 287 (1961).

[40] G. Baym, Phys. Rev. 127, 1391 (1962).

[41] W. Fu and S. Sachdev, Phys. Rev. B 94, 035135 (2016).

[42] For fillings $\gtrsim 0.81$ and $\lesssim 0.19$, the $q=4$ SYK model has a first-order phase transition to a nearly free fermion phase at temperatures $T \ll U$ [44], and we are hence unable to explore those regions numerically.

[43] M. Calandra and O. Gunnarsson, Europhys. Lett. 61, 88 (2003).

[44] T. Azeyanagi, F. Ferrari, and F. I. S. Massolo, Phys. Rev. Lett. 120, 061602 (2018).

Correction: Support information was missing in the Acknowledgment section and has been inserted. 